\title{
The Modern Profile of Tuberculosis: Developing the TB Social Survey to understand contemporary social patterns in tuberculosis
}

\section{Grace A. Noppert PhD ${ }^{1,2}$ (D) | Philippa Clarke PhD ${ }^{2,3}$}

${ }^{1}$ The Center for the Study of Aging and Human Development, Duke University, Durham, NC, USA

${ }^{2}$ Department of Epidemiology, School of Public Health, University of Michigan, Ann Arbor, MI, USA

${ }^{3}$ Institute for Social Research, University of Michigan, Ann Arbor, MI, USA

\section{Correspondence}

Grace Noppert, The Center for the Study of Aging and Human Development, Duke University, Durham, NC, USA.

Email: grace.noppert@duke.edu

\section{Funding information}

This study was funded by a research grant from the Rackham Graduate School at the University of Michigan. Dr. Noppert also received partial support from the Duke Center for the Study of Aging and Human Development, NIA grant T32-AG000029-40.

\begin{abstract}
Social disparities in tuberculosis have been documented for decades, yet to date there has not been a comprehensive study to examine the contemporary causes of these disparities. Local public health departments, and particularly public health nursing staff are charged with delivering directly observed therapy to individuals with tuberculosis disease. As a result of the frequency and duration of treatment, practitioners delivering therapy are often well-acquainted with the lives and challenges of their constituents. Thus, through these practitioners there exists a deep repository of knowledge on the drivers of social disparities in tuberculosis disease. Partnering with local public health departments, we developed a survey instrument aimed at understanding the social profile of individuals with tuberculosis disease in metropolitan Detroit, Michigan. We discuss the development and implementation of the survey instrument as well as challenges in developing partnerships between academic researchers and local public health practitioners. This study can serve as a framework for both academic researchers and public health practitioners interested in addressing social disparities in infectious disease.
\end{abstract}

\section{KEYWORDS}

health status disparities, nursing, public health practice, social determinants of health, tuberculosis

\section{1 | INTRODUCTION}

\subsection{Social disparities in tuberculosis}

After decades of decline, the incidence of tuberculosis (TB) in the United States has leveled off with no signs of decline in recent years (Salinas et al., 2016). What remains are persistent and entrenched disparities in TB incidence along lines of social disadvantage. There is a long history of social disparities in TB in the United States. Despite the ubiquity of TB exposure during the 19th and early-20th centuries, TB disproportionately burdened socially disadvantaged populations, particularly racial/ethnic minorities, immigrants, and those in poverty. Social disparities in TB incidence continue to persist in the United
States today. While the roots of these disparities are decades old, the contemporary causes are not well understood.

Currently, TB still disproportionately affects the foreign-born, racial/ethnic minorities, the poor, those living in urban environments, the homeless, and the incarcerated (Alami et al., 2014; Driver et al., 2007; Ellis et al., 2002). The incidence of recently transmitted TB in the United States is six times higher in Blacks compared to Whites (Noppert et al., 2017). Disparities are even larger in specific states, such as Michigan. From 2004 to 2012, the average incidence of recently transmitted TB in Michigan was nearly twenty times greater in Blacks than Whites (Noppert et al., 2017). Similarly, the average incidence of reactivation of latent TB infection (LTBI) in Michigan was 19 
times greater in the foreign-born compared to the U.S.-born (Noppert et al., 2017).

Despite evidence of significant social disparities, reports on the trends and distribution of TB in the United States are based predominantly on surveillance data, which lack the detailed individual-level data to fully understand the reasons for these persistent disparities. While there is an acknowledgement that TB disparities exist among certain subpopulations (i.e., among racial/ethnic minorities and socioeconomically disadvantaged individuals), there is a lack of research on other social factors that could help to identify the underlying mechanisms by which these populations remain at greater risk. The purpose of this study was to develop a survey instrument aimed at systematically collecting detailed data on the social experience of individuals with TB.

\subsection{Lack of data to understand the reasons for social disparities}

The paucity of research on the mechanisms underlying the social disparities in TB is driven, in part by, a lack of detailed data on the social and economic circumstances of current TB patients. In her 2000 paper, Acevedo-Garcia put forth a framework for understanding how one social process, residential segregation, may impact the epidemiology of TB (Acevedo-Garcia, 2000). However, the extent to which we can operationalize this framework across contexts, or extend it to include other social processes is hampered by the lack of data of this nature. Moreover, there are increasing calls for those in infectious disease research to employ the tools of social epidemiology to better address social disparities in infectious diseases (Cohen, Wilson, \& Aiello, 2007; Noppert, Kubale, \& Wilson, 2016). Our ability to do so, however, is dependent on the collection of contemporary data on the social profile of TB patients.

Much of what we know about the social experience of individuals with TB in the United States is derived from evidence (both formal and anecdotal) that is decades old. Current sociodemographic data are limited to what is available on the patient chart and the "Report of a Verified Case of TB" (RVCT) form produced by the Centers for Disease Control and Prevention (CDC) (Centers for Disease Control and Prevention, n.d.). These data are often based on clinician observation and are mainly focused on collecting data on known TB risk factors. To date, very few studies have been able to ascertain detailed sociodemographic data on TB patients. However, these types of data exist informally at the local public health level, particularly among those charged with carrying out directly observed therapy (DOT).

DOT is a central tenet to the care of individuals with TB in the United States (Centers for Disease Control and Prevention, 2013). To carry out DOT, a health practitioner, most often a public health nurse, must observe the individual with TB taking the prescribed medication on a regular basis. Given the long duration of TB drug therapy (typically 4-6 months) and the regularity with which medications must be taken, the practitioner often inadvertently becomes involved in the patient's life.
Thus, while academic researchers may lack the requisite data to bridge the gaps between observed disparities and the drivers of those disparities, public health practitioners are keenly aware of the complex interplay between TB disease and the social and economic status of individuals. Yet, this knowledge has not been collected in a systematic way that would allow for wider use by both the research community and by those designing policies and interventions for TB control.

\section{3 | Developing a survey instrument to understand social disparities}

To address the lack of data on social and economic factors that could be underlying persistent TB disparities, we developed a survey instrument aimed at systematically collecting detailed data on the social experience of individuals with TB. We pilot tested the instrument in metropolitan Detroit, Michigan, an area with ongoing incidence of TB. Our study had two primary aims: one, to develop a survey instrument that could be utilized by local public health agencies; and two, to gather detailed data on the social and economic profile of TB patients in metropolitan Detroit. Our purpose in this paper is to discuss the process of developing the survey instrument; the study infrastructure for implementation of the instrument; and challenges in developing partnerships between academic researchers and local public health practitioners. Although the survey is ongoing and data continue to be collected, our goal is that this survey instrument can ultimately be implemented at the national level to yield important insights into the modern TB epidemic in the United States, and facilitate the effective allocation of resources to those most at risk.

\section{2 | METHODS}

\section{1 | Target population and study site recruitment}

Our target population was individuals with TB in metropolitan Detroit. From 2007 to 2012, the incidence of TB disease in Detroit was 58\% greater compared to outside of Detroit. The three health departments recruited for participation in this study accounted for $55 \%$ of all TB cases in Michigan from 2007 to 2012 (data taken from MDHHS surveillance data).

In order to recruit study participants from this population, we partnered with three local public health agencies carrying out DOT in this area: Oakland County Division of Health, Wayne County Health Department, and the Physicians Group at Wayne State University. (one of the Detroit City Health Departments) The three study sites were chosen in collaboration with the TB control staff at the Michigan Department of Health and Human Services (MDHHS). MDHHS staff identified local public health agencies who served the largest patient populations in metropolitan Detroit. Once identified, the research team at the University of Michigan contacted the local public health agencies and invited each site to participate in the study.

Our collaborations were formalized after months of rapport building between the research team at the University of Michigan and practitioners at the local level. Our formal collaboration with each health 
TABLE 1 Major components of the TB Social Survey

\begin{tabular}{|c|c|}
\hline Survey section & Specific variables \\
\hline \multirow[t]{4}{*}{ Part 1: Demographics } & Race/Ethnicity \\
\hline & Gender \\
\hline & Marital status \\
\hline & Education \\
\hline \multirow[t]{4}{*}{ Part 2: Life before TB } & Self-rated health \\
\hline & $\begin{array}{l}\text { Alcohol usage, past and current smoking } \\
\text { behavior, physical activity }\end{array}$ \\
\hline & $\begin{array}{l}\text { Health care-seeking behaviors, barriers to } \\
\text { seeking health care }\end{array}$ \\
\hline & $\begin{array}{l}\text { Locations frequented throughout the } \\
\text { community }\end{array}$ \\
\hline \multirow[t]{8}{*}{ Part 3: Current status } & $\begin{array}{l}\text { Housing (history of homelessness, } \\
\text { transience in housing, crowded housing } \\
\text { condition) }\end{array}$ \\
\hline & Neighborhood condition \\
\hline & $\begin{array}{l}\text { Health insurance, barriers to having health } \\
\text { insurance }\end{array}$ \\
\hline & Employment \\
\hline & $\begin{array}{l}\text { Income (including ability to make monthly } \\
\text { bill payments }\end{array}$ \\
\hline & Subjective social status \\
\hline & Access to social support \\
\hline & Food security \\
\hline
\end{tabular}

department also included joint IRB approvals between the MDHHS, the University of Michigan and each individual health department. The study team at the University of Michigan provided a 1-day training in survey administration and human subjects' protection at each study site.

\section{2 | Study design}

This project is a pilot study, the purpose of which was to develop a survey instrument to be used in community public health settings to ascertain the social status of individuals with active TB disease. In the United States, TB is a notifiable disease whose treatment is covered by the state health resources. As such, each case of TB disease is reported to the local health department (usually county-level) who then facilitates treatment. We decided the survey instrument could most efficiently be delivered by utilizing the existing infrastructure of local public health agencies, which have both a record of all existing cases in their catchment area as well as regular contact with such individuals. The survey is administered by the health care workers overseeing DOT to persons with TB.

The survey is given at one time point during the drug therapy period. When planning for survey administration, it was decided in collaboration with the health departments, that surveys should be administered once rapport has been built between the individual with TB disease and the health care professional, a minimum of 4 weeks into therapy.

\section{3 | Survey participant recruitment}

Inclusion criteria for participation in the study included: individuals with active TB disease identified at one of the three participating metropolitan Detroit health departments; any individual currently in treatment or who had completed treatment in the last year; ability to speak and understand English; and over the age of 18. Health care professionals administering DOT invited each individual to participate in the study. The health care professional explained the purpose of the study as well as any risks associated with participation in the study. Written informed consent was obtained from each individual participating in the study. Survey participants were offered a one-time $\$ 20$ incentive for participation in the study in the form of a gift card to a local grocery store.

\section{4 | Survey development}

The process of developing a survey instrument to fit the needs of this specific patient population was an iterative process spanning months, and involving many different parties. The survey needed to accomplish three main objectives: 1) collect detailed sociodemographic variables relevant to the lives of persons with TB disease in a standardized format; 2) collect these data in a manner feasible for the participants, study team, and the health department staff to carry out; and 3) create buy-in from both the state and local health departments to carry out the study.

In designing the survey instrument, we collated months of information that had been gathered from meetings with state and local TB control staff. Given the regularity of contact and length of treatment for TB disease, local public health agencies often have a deep repository of invaluable anecdotal data on the social and economic lives of their constituents. To leverage this knowledge, we spent months talking with local TB control staff by sitting in cohort reviews of the current TB patients receiving treatment and through one-on-one conversations with the staff. We then used this knowledge to begin constructing the survey instrument.

We also examined the RVCT form to identify either components missing from the form or questions that were based on clinician observation rather than patient self-report. We used this information to identify the key question themes that should be addressed by the survey.

Where possible we used questionnaire items from national surveys and major cohort studies that were valid and reliable to begin compiling potential questions. These studies included: the Jackson Heart Study (Taylor et al., 1999), the Detroit Neighbor Health Study (Goldmann et al., 2011), the Multi-Ethnic Study of Atherosclerosis (Bild et al., 2002), the U.S. Health and Retirement Study (Juster \& Suzman, 1995), and the Americans' Changing Lives Study (Lantz, House, Mero, \& Williams, 2005). However, for those themes for which we could not find an adequate question, we developed our own.

The purpose of the survey instrument was to understand the current social profile of TB patients in terms of their basic demographics, 
TABLE 2 Preliminary results of the TB Social Survey. The following table describes the demographic characteristics of the preliminary study sample $(N=23)$.

\begin{tabular}{|c|c|c|}
\hline Variable & M & $S D$ \\
\hline Age (M) & 42.4 & 17.38 \\
\hline \multicolumn{3}{|l|}{ Missing $=3$} \\
\hline & $N$ & $\%$ \\
\hline \multicolumn{3}{|l|}{ Nativity } \\
\hline U.S.-born & 9 & 39.13 \\
\hline Foreign-born & 11 & 47.83 \\
\hline Missing & 3 & 13.04 \\
\hline \multicolumn{3}{|l|}{ Race/ethnicity } \\
\hline Black/African-American & 7 & 30.43 \\
\hline White & 6 & 26.09 \\
\hline Asian Indian & 5 & 21.74 \\
\hline Other & 5 & 21.74 \\
\hline Middle Eastern/North African descent & 6 & 26.09 \\
\hline Hispanic descent & 2 & 8.70 \\
\hline \multicolumn{3}{|l|}{ Gender } \\
\hline Male & 8 & 34.78 \\
\hline Female & 15 & 65.22 \\
\hline \multicolumn{3}{|l|}{ Marital status } \\
\hline Married & 10 & 43.48 \\
\hline Separated & 1 & 4.35 \\
\hline Divorced & 2 & 8.70 \\
\hline Widowed & 3 & 13.04 \\
\hline Living with a partner & 2 & 8.70 \\
\hline Single & 5 & 21.74 \\
\hline \multicolumn{3}{|l|}{ Education } \\
\hline Some high school & 3 & 13.04 \\
\hline High school/GED & 7 & 30.43 \\
\hline Some college & 5 & 21.74 \\
\hline Bachelor/associate & 5 & 21.74 \\
\hline Other professional degree & 2 & 8.70 \\
\hline Missing & 1 & 4.34 \\
\hline
\end{tabular}

Results based on preliminary analyses of 23 individuals.

social status, and economic status. We also wanted to gather evidence on what their social profile was before they were diagnosed with TB. We did this explicitly with questions asking them to recall their status before diagnosis. However, in some cases we also assumed that current life circumstances could be used as a proxy measure for their conditions before TB diagnosis. We hypothesized that social factors may have a role in patterning not only one's risk of progressing to active TB disease, but also the probability that one is exposed to Mycobacterium tuberculosis (MTB), the pathogen causing TB disease.

The final survey, entitled the "TB Social Survey" was split into three main parts: demographics, life before TB, and current status (Table 1). In the demographics section of the survey, participants were asked about their race/ethnicity status, gender, marital status, and educational status. The next section was composed of questions designed to ascertain details of the participants' life before TB. Included in these were questions about general health status, health behaviors, including health-seeking behaviors, and locations frequented in the community. Asking about locations frequented in the community was an attempt to understand the places where an individual may have been exposed to the MTB pathogen. Finally, the largest section of the survey was devoted to understanding features of the individuals' current life. These questions encompassed housing conditions (e.g., history of homelessness, transient housing, and living conditions); neighborhood conditions; current health insurance; employment and income status (including ability to pay bills); subjective social status; and food security. The final survey includes 30-questions, and takes approximately 20-25 min to administer. We pretested the survey for 3 months before survey launch. Pretesting was done among ten individuals without TB, including eight women and two men, with a range of incomes. Nine of the ten individuals were $30-40$ years of age. Further, before survey launch each health department gave feedback on the survey which was incorporated into the final survey. The final survey instrument was provided to each health department before the formal launch of the study. IRB approval was obtained for the collection of the survey data at each study site and at MDHHS. For the complete survey instrument, see Supplemental Digital Content S1.

\section{3 | PRELIMINARY FINDINGS}

\subsection{Characteristics of the study population}

To date, a total of 26 individuals have been recruited to participate in the study; 23 completed the survey for a survey completion rate of $88.46 \%$. Of the 23 participants, $65 \%$ were from the Wayne County Health Department; $22 \%$ from the Oakland County Division of Health, and $13 \%$ from the Physicians Group at Wayne State University.

Characteristics of these participants are presented in Table 2. Of the 23 individuals with completed surveys, 39\% were U.S.-born, $48 \%$ foreign-born and $13 \%$ were missing data on nativity (Table 2). The participants were 30\% Black/African American, 26\% White, 22\% Asian Indian, and $22 \%$ some other race. Additionally, $26 \%$ of the sample identified as Middle Eastern/North African descent. Over half (65\%) of the participants were female and the mean age was 42.4 years. The study is ongoing but will likely continue to replicate the sociodemographic patterns observed to date.

Responses to a subset of survey questions are given in Table 3. We asked participants a series of questions about their health before their diagnosis of TB. Regarding their health before diagnosis, 91\% of participants reported that they were in good or excellent health and $30 \%$ reported having smoked cigarettes before their diagnosis. Finally, before TB the majority (70\%) of participants reported engaging in active sports or exercise at least once per week.

We also asked participants about their current life including income, living conditions, and their neighborhood environment. While $35 \%$ of the sample refused to report their income, $17 \%$ reported an 
income less than $\$ 15,000 ; 13 \%$ reported an income between $\$ 15,000$ and $\$ 30,000 ; 13 \%$ reported an income between $\$ 30,000$ and $\$ 75,000$; and $4 \%$ reported an income greater than $\$ 75,000$. Of the sample, $39 \%$ reported some difficulty making monthly bill payments. Over a quarter (26\%) of participants reported some difficulty making food last until there was money to buy more.

When asked about their current living conditions, $96 \%$ of participants reported having a permanent address; $4 \%$ reporting living in a public housing project. Moreover, $87 \%$ of the sample rated the condition of where they lived as good or excellent.

Item nonresponse was notable in our study for the income question. The section of the survey regarding income was placed at the end of the survey. Thirty-five percent of participants refused to answer this question at all. Seventeen percent of participants reported not knowing their totally household yearly income.

The data presented here are preliminary results. Data collection will continue through 2017. We expect to have approximately 70 participants total from all three health departments.

\section{4 | INSIGHTS AND FUTURE IMPLICATIONS}

As far as we are aware, this is the first standardized survey instrument developed to collect data on the social profile of TB. The process of carrying out the study as well as the preliminary results offers important insights into the sociodemographic profile of TB patients in metropolitan Detroit. Ultimately, we hope the process of ascertaining detailed sociodemographic information on TB patients will be replicated in other health departments both in Michigan, and nationally. In addition, we hope our findings will be used to guide TB control efforts, particularly in metropolitan Detroit where limited resources need to be allocated efficiently and effectively.

Our preliminary results allow us to begin to understand the modern sociodemographic profile of individuals with TB in the United States. For instance, asking about individuals' living conditions, ability to make bill payments, and food availability gives us critical information on the social vulnerability of an individual. A large portion of participants (39\%) have thus far reported some difficulty making monthly bill payments and $26 \%$ reported some difficulty making food last. These data suggest that there may be underlying insecurity with accessing basic resources. This insecurity may have implications for overall health status likely increasing probability of developing active TB disease once exposed to MTB.

These findings may also give us important insights into how we might best design interventions that improve the health conditions of individuals with TB. For example, it is likely that many of those receiving treatment for TB disease may also benefit from assistance in getting access to food. While many health departments delivering TB care work to address these needs, our findings can provide evidentiary support for increased funding for such interventions.

We encountered several key methodological challenges in this study: namely, nonparticipation in the survey and item nonresponse for particular questions. We examined reasons for both occurrences in order to design strategies to mitigate the resulting bias in the future as well as to understand how the survey should be modified in future iterations.

Nationally, survey nonparticipation is increasing (Massey \& Tourangeau, 2013). This increase is most notable in cross-sectional surveys in which researchers do not have the benefit of offering consistent financial incentives over time (Massey \& Tourangeau, 2013). Further, nonparticipation is more likely to occur among the disadvantaged: minorities, males, urban residents, singe persons, the poor, and those with fewer social ties and attachments (Schoeni, Stafford, McGonagle, \& Andreski, 2013)-precisely the population we were most interested in studying.

Massey and Tourangeau give three primary reasons for nonparticipation: noncontact-interviewers being unable to make contact with potential participants; refusals-contact is made but participants decline to participate; and a residual category encompassing such reasons as too busy, sick, participant/interviewer differences (Massey \& Tourangeau, 2013). Refusals consistently account for the largest proportion of unit nonresponse-typically 60\%-65\% (Brick \& Williams, 2012). In our study, noncontact was less of an issue given that potential participants were individuals enrolled in TB treatment. Refusals were, however, the primary reason for nonresponse. Since the interviewer has an established rapport with participants prior to the administration of the survey, we were able to gather anecdotal evidence for the reasons for nonresponse. Most of our refusals were among foreign-born persons who expressed fear of their data being transmitted to the U.S. government. The health care workers noted that the rate of nonparticipation from foreign-born persons seemed to increase in times when contemporary immigration fears and policies were amplified in the public. They also noted this seemed more of an issue with newly arrived immigrants as opposed to those with established residency in the United States. Thus, despite the one-time financial incentive we offered survey participants, the risks and threats to confidentiality outweighed the financial incentive for this particular population.

Item nonresponse, or refusing to answer specific questions, was also a methodological challenge we faced. Nationally, rates of item nonresponse are also increasing, particularly for sensitive questions such as those dealing with income and receipt of government benefits (Meyer, Mok, \& Sullivan, 2015). Meyer et al. (2015) hypothesize three reasons for item nonresponse: refusal to answer, inability to answer, or failure of the interviewer to accurately record the participant response. Item nonresponse was notable in our study in terms of the income question. Over $35 \%$ of participants refused to answer this question at all. All three of the above factors certainly influenced participants' willingness to answer the income question in our study. Some participants simply refused to answer the question while others cited a fear of disclosure of private information to the U.S. government. Others were simply unable to answer the question either because of language difficulties or being a member of a family unit in which he or she is not privy to such information. 
TABLE 3 Preliminary results of the TB Social Survey. The following table describes the participant responses to select questions from the TB Social Survey $(N=23)$.

Variable N $\quad$ \%

"Before you had TB, would you say your health was excellent, good, fair, or poor compared with other people your age?"

$\begin{array}{lrr}\text { Excellent } & 10 & 43.48 \\ \text { Good } & 11 & 47.83 \\ \text { Fair } & 2 & 8.70 \\ \text { "Did you ever drink beer, wine, or liquor?" } & \\ \text { Yes } & 8 & 34.78 \\ \text { No } & 15 & 65.22\end{array}$

"Did you smoke cigarettes before you had TB?"

$\begin{array}{crr}\text { Yes } & 7 & 30.43 \\ \text { No } & 15 & 65.22 \\ \text { Missing } & 1 & 4.35 \\ \text { "Have you ever smoked?" } & & \\ \text { Yes } & 6 & 26.09 \\ \text { No } & 13 & 56.52 \\ \text { Missing } & 4 & 17.39\end{array}$

"Before your diagnosis of TB, how often did you engage in active sports or exercise?"

\begin{tabular}{lcr} 
Never & 5 & 21.74 \\
\hline 1 × per week & 2 & 8.70 \\
Once a week & 6 & 26.09 \\
Multiple times per week & 4 & 17.39 \\
Almost every day & 6 & 26.09 \\
"Do you have a permanent address?" & \\
Yes & 22 & 95.65 \\
No & 0 & \\
Missing & 1 & 4.35 \\
"Do you live in a public housing project?" & \\
Yes & 1 & 4.35 \\
No & 22 & 95.65 \\
"How would you rate the condition of where you live?" & \\
Excellent & 13 & 56.52 \\
Good & 7 & 30.43 \\
Fair & 2 & 8.70 \\
Poor & 1 & 4.35 \\
\hline
\end{tabular}

"How would you rate the quality of your neighborhood, that is the area within 2-3 blocks of where you live?"

$\begin{array}{lrr}\text { Excellent } & 9 & 39.13 \\ \text { Good } & 10 & 43.48 \\ \text { Fair } & 2 & 8.70 \\ \text { Poor } & 2 & 8.70\end{array}$

"How difficult is it for you to make your monthly bill payments?"

\begin{tabular}{lrc} 
Not difficult & 12 & 52.17 \\
Somewhat & 7 & 30.43 \\
Very & 1 & 4.35 \\
\hline
\end{tabular}

TABLE 3 (Continued)

\begin{tabular}{|c|c|c|}
\hline Variable & $N$ & $\%$ \\
\hline Extremely & 1 & 4.35 \\
\hline Refused & 0 & \\
\hline Missing & 2 & 8.70 \\
\hline \multicolumn{3}{|c|}{ Total household yearly income (\$) } \\
\hline$<15,000$ & 4 & 17.39 \\
\hline $15,000-30,000$ & 3 & 13.04 \\
\hline $30,000-75,000$ & 3 & 13.04 \\
\hline$>75,000$ & 1 & 4.35 \\
\hline Do not know & 4 & 17.39 \\
\hline Refused & 8 & 34.78 \\
\hline
\end{tabular}

"How difficult is it for you to make food last until you have money to buy more?"

$\begin{array}{lrr}\text { Not difficult } & 17 & 73.91 \\ \text { Somewhat difficult } & 6 & 26.09\end{array}$

"Have you had to cut down the number or size of meals because of money?"

$\begin{array}{lrr}\text { Yes } & 2 & 8.70 \\ \text { No } & 21 & 91.30\end{array}$

Results based on preliminary analyses of 23 individuals.

The third explanation for item nonresponse, that of the failure of the interviewer to accurately record a response, applies both to the income-related questions in our survey as well as to a number of other sensitive questions. In some instances, the response given to a certain question was too obscure and time-consuming for the health care worker to disentangle and indicate on the survey. In these cases, the health care workers reported they would skip recording a response to the question. We also saw a related issue in our survey-failure of the interviewer to ask the question. In our survey training, we gave interviewers the autonomy to decide whether or not to ask a question based on verbal and nonverbal cues from the participant. The health care workers indicated that questions of a sensitive nature such as income, housing, and social standing sometimes visibly upset the participant. On such occasions, the health care worker would simply not continue with the question or its related questions. One step we would like to implement in future iterations of the survey is the addition of an item on each question indicating if the interviewer skipped asking the question altogether. This may help us disentangle refusal to answer a question, inability to record a response, and failure to ask a question from one another.

\section{1 | Lessons learned and next steps}

In addition to the strengths and limitations of the study itself, there were several lessons we learned with regards to developing collaborations between academia and local public health agencies. One of the most critical lessons was the need for buy-in from all stakeholders. The local public health agencies were keenly aware of the needs and challenges of their patient population and thus were able 
to give critical feedback on the survey instrument itself as well as the logistics of carrying out the study. Throughout the duration of the study, we found it helpful to continually cultivate the relationships with the local public health agencies, particularly the public health nursing staff. Often these practitioners are working within resource constrained environments and may have many demands on their time. The study team held a lunch meeting twice per year for each local public health agency in order to provide a time and space for the staff to give feedback on the progress of the study, to solve any issues, and to continue to build rapport between the two stakeholders.

Launching the study initially required much more time than we anticipated due to having to obtain multiple IRB approvals across study sites. In the future, we would allocate much more time for this process and set up structures to deal with any potential issues that may arise during each IRB approval process.

There are several major strengths to this study that can offer direction to future studies. First, to our knowledge there has not been a study such as this conducted in the modern U.S. context. This is one of the first studies to utilize a survey instrument to collect detailed sociodemographic information on individuals with TB. It is our hope that the data we obtain from our survey can provide insights into why the TB epidemic has continued in the United States, and moreover why disparities in TB incidence continue to persist.

Another key strength of our study is that it allows participants to self-report their sociodemographic characteristics as opposed to the previous reliance on health care worker observation on the RVCT form. How an individual identifies oneself can be much more indicative of their social experience than how a health care worker may identify them.

Our study has also developed a framework for collaborations between state/local health departments and academia around issues of TB control. Such collaborations are mutually beneficial and can result in data that can better inform both TB control and TB research. For example, the health departments that participated in this study are utilizing the data from this study as well as input from the researchers to develop grant applications for increased funding for their TB control programs. The health departments also noted the benefit of having more detailed data to report out to the state and city health officials; such data highlight the need for sustained funding for infectious disease control programs, such as TB control. Finally, the health departments have articulated a desire to apply this methodology to other infectious diseases under the jurisdiction of the local health department.

There are also several limitations to our study. While there are many benefits to the survey being administered by health care workers (i.e., rapport, trust), some participants were still fearful of the consequences of their participation for the quality of their care. The health care workers consistently reported that nativity was an important factor influencing how questions were interpreted. For example, foreign-born participants questioned whether they should report their highest education based on U.S. metrics or based on the years of education in their country of origin. Given the high proportion of foreign-born persons in the TB population, it would be beneficial to tailor certain questions to better fit the needs of the foreign-born population.

Further, the results of this study are prone to recall bias, particularly for the series of questions in which participants were asked to recall the time before they had TB. For some participants, this may have been up to 1 year ago. Moreover, because of recall bias, we suspect that participants may have remembered their time before TB as better than it was in comparison to their current status.

Finally, since this is a pilot study, we do not have a control group without TB. We cannot compare our study results with a TB-free population which would allow us to quantify the degree to which certain variables put individuals at risk for TB. However, we believe having the baseline sociodemographic characteristics of this population will make such studies possible in the future.

In spite of these limitations, this study provides an instrument to systematically collect data on the social experience of TB patients. These data are critical to informing interventions to better address the TB epidemic in the United States.

In future iterations of the study, we would like to develop strategies to better ascertain information from individuals who feel atrisk-such as foreign-born persons. One way to do this might be to change the timing of survey administration. Many of the health care workers noted ongoing health care-related encounters with patients after treatment completion. Post-treatment health care encounters may present a more comfortable time for at-risk persons to be administered the survey both because they have increased rapport with the health care worker and they are no longer required to undergo treatment. Another way to ensure participation of at-risk individuals may be to have a patient advocate present for survey administration; someone such as a trusted family member and/or social worker. However, this may also diminish the individuals' likelihood of responding to certain questions.

Moreover, for public health agencies seeking to implement this tool, linking the data collected on the survey to treatment outcomes may be beneficial. For example, understanding if treatment adherence is tied to any particular social vulnerability may be insightful for the health department staff. Treatment completion is of critical importance to both the health of the individual and reducing the development of drug-resistant forms of the MTB pathogen.

\section{2 | Implications for public health practice}

The findings of this study can aid public health practitioners at both the local and national levels. At the local public health level, these data could provide those in TB control a better understanding of which populations continue to be at-risk for TB such that control strategies could be tailored accordingly. Implementing this survey at public health departments nationwide could provide critical data on the current landscape of TB in the United States. Such data could provide insight in how to best allocate limited resources and design interventions that would move the United States closer to TB elimination. 


\section{ACKNOWLEDGEMENTS}

The authors are grateful for the continued support of Dr. Peter Davidson and the Michigan Department of Health and Human Services. The support of Dr. Davidson has been invaluable in building the infrastructure for this project, including building relationships with the local health departments. Additionally, the authors are immensely grateful to the Oakland County Health Department, the Wayne State Physicians Group, and the Wayne County Health Department without whom this study would not have been possible. Their expertise and insights have been invaluable to the development of this study. Specifically, the authors would like to acknowledge Pamela Hackert, MD JD MPH; Janice Taylor, RN; and Laura Frederick, RN for the countless hours spent with the study team, educating them on the on-the-ground experience of TB control. This education and support has been instrumental to the study and is most appreciated. The authors are also grateful to Dr. Zhenhua Yang and Dr. Mark Wilson for their continued support for this project. Their expertise has been invaluable to the authors' understanding of the pathophysiology of TB and has served as a foundation for interpreting the findings of this study.

\section{ORCID}

Grace A. Noppert (iD http://orcid.org/0000-0002-2040-960X

\section{REFERENCES}

Acevedo-Garcia, D. (2000). Residential segregation and the epidemiology of infectious diseases. Social Science \& Medicine, 51(8), 1143-1161.

Alami, N. N., Yuen, C. M., Miramontes, R., Pratt, R., Price, S. F., Navin, T. R., \& Centers for Disease Control and Prevention. (2014). Trends in tuberculosis-United States, 2013. MMWR. Morbidity and Mortality Weekly Report, 63(11), 229-233.

Bild, D. E., Bluemke, D. A., Burke, G. L., Detrano, R., Diez-Roux, A. V., Folsom, A. R., ... Nelson, J. C. (2002). Multi-Ethnic Study of Atherosclerosis: Objectives and design. American Journal of Epidemiology, 156(9), 871-881.

Brick, J. M., \& Williams, D. (2012). Explaining rising nonresponse rates in cross-sectional surveys. The Annals of the American Academy of Political and Social Science, 645(1), 36-59. https://doi. org/10.1177/0002716212456834

Centers for Disease Control and Prevention. (2013). Core curriculum: What the clinician should know - TB. Retrieved from http://www.cdc.gov/tb/ education/corecurr/default.htm

Centers for Disease Control and Prevention. (n.d.). CDC | TB | Report of Verified Case of Tuberculosis (RVCT). Retrieved from http://www.cdc. gov/TB/programs/rvct/default.htm

Cohen, J. M., Wilson, M. L., \& Aiello, A. E. (2007). Analysis of social epidemiology research on infectious diseases: Historical patterns and future opportunities. Journal of Epidemiology and Community Health, 61(12), 1021-1027. https://doi.org/10.1136/jech.2006.057216
Driver, C. R., Kreiswirth, B., Macaraig, M., Clark, C., Munsiff, S. S., Driscoll, J., \& Zhao, B. (2007). Molecular epidemiology of tuberculosis after declining incidence, New York City, 2001-2003. Epidemiology and Infection, 135(4), 634-643.

Ellis, B. A., Crawford, J. T., Braden, C. R., McNabb, S. J. N., Moore, M., \& Kammerer, S. (2002). Molecular epidemiology of tuberculosis in a sentinel surveillance population. Emerging Infectious Diseases, 8(11), 1197-1209.

Goldmann, E., Aiello, A. E., Uddin, M., Delva, J., Koenen, K., \& Grant, L. M. (2011). Pervasive exposure to violence and posttraumatic stress disorder in a predominantly African American Urban Community: The Detroit Neighborhood Health Study. Journal of Traumatic Stress, 24(6), 747-751.

Juster, F. T., \& Suzman, R. (1995). An overview of the health and retirement study. Journal of Human Resources, 30, S7-S56. Retrieved from https:// hrs.isr.umich.edu/documentation

Lantz, P., House, J. S., Mero, R. P., \& Williams, D. R. (2005). Stress, life events, and socioeconomic disparities in health: Results from the Americans' changing lives study. Journal of Health and Social Behavior, 46(3), 274-288.

Massey, D. S., \& Tourangeau, R. (2013). Where do we go from here? Nonresponse and social measurement. The Annals of the American Academy of Political and Social Science, 645(1), 222-236.

Meyer, B., Mok, W., \& Sullivan, J. (2015). Household surveys in crisis. Retrieved from http://harris.uchicago.edu/sites/default/files/ JEPSurveys02042015.pdf

Noppert, G., Kubale, J., \& Wilson, M. (2016). Analyses of infectious disease patterns and drivers largely lack insights from social epidemiology: Contemporary patterns and future opportunities. Journal of Epidemiology and Community Health, 71(4), 350-255.

Noppert, G. A., Wilson, M. L., Clarke, P., Ye, W., Davidson, P., \& Yang, Z. (2017). Race and nativity are major determinants of tuberculosis in the U.S.: Evidence of health disparities in tuberculosis incidence in Michigan: 2004-2012. BMC Public Health, 17, 538.

Salinas, J. L., Mindra, G., Haddad, M. B., Pratt, R., Price, S. F., \& Langer, A. (2016). Leveling of tuberculosis incidence-United States, 2013-2015. MMWR. Morbidity and Mortality Weekly Report, 65, 273-278.

Schoeni, R. F., Stafford, F., McGonagle, K. A., \& Andreski, P. (2013). Response rates in national panel surveys. The Annals of the American Academy of Political and Social Science, 645(1), 60-87.

Taylor, H. A. Jr, Burchfield, C., Fletcher, B., Jones, D., Mehrotra, B., \& Ruffin, J. (1999). The Jackson heart study. Association of Black Cardiologists: Digest of Urban Cardiology, 6, 27-33.

\section{SUPPORTING INFORMATION}

Additional Supporting Information may be found online in the supporting information tab for this article.

How to cite this article: Noppert GA, Clarke P. The Modern Profile of Tuberculosis: Developing the TB Social Survey to understand contemporary social patterns in tuberculosis. Public Health Nurs. 2018;35:48-55.

https://doi.org/10.1111/phn.12372 